\title{
DIFICULTADES DE APRENDIZAJE DE LOS CONCEPTOS DE CARGA Y DE CAMPO ELÉCTRICO EN ESTUDIANTES DE BACHILLERATO Y UNIVERSIDAD
}

\author{
FURIÓ, C. ${ }^{1}$ y GUISASOLA, J. ${ }^{2}$ \\ Departamento de Didáctica de la Ciencias Experimentales. Universidad de Valencia. \\ 2 Departamento de Física Aplicada I. Universidad del País Vasco.
}

\begin{abstract}
SUMMARY
This article tries to analyse what the students' principal learning difficulties on the concept of charge and electric field are. In this way, we have supposed certain parallels between learning difficulties in these concepts and the problems in their formation throughout history. We have carried out a short description of principal epistemological problems, and a design based on a questionnaire containing open questions and personal interviews of secondary and University students. The results obtained seem to indicate that the students have problems to interpret electrification phenomena, which are not easy to explain by a hydrostatic model of electric charge. Likewise, we have verified that few students significantly use the concept of electric field.
\end{abstract}

\section{INTRODUCCIÓN}

Las dificultades de aprendizaje de las principales nociones de electricidad, tales como potencial eléctrico o corriente eléctrica, están siendo investigadas, extensamente, en el nivel de la enseñanza secundaria. Sin embargo, estos trabajos se han centrado más en el dominio de la electrocinética (Duit, 1993) y, en particular, en los circuitos eléctricos sin que normalmente se establezcan relaciones con la electrostática y, especialmente, con el concepto de campo eléctrico, que es indispensable para entender el movimiento de cargas en circuitos eléctricos (Eylon y Ganiel,1990; Viennot y Rainson, 1992).

Muy probablemente, estos problemas de aprendizaje que se presentan en la naturaleza eléctrica de la materia $y$, en particular, en el concepto de campo eléctrico, puedan atribuirse, por un lado, a ideas previas de los estudiantes derivadas de un análisis «superficial» de las experiencias sensoriales relativas a electrostática (frota- miento de un bolígrafo y atracción de papelitos, electrización de la carrocería de un coche al desplazarse o de la pantalla de la televisión...) y, por otro, a una deficiente familiarización de los estudiantes en los métodos y contenidos de la ciencia (Gil y Carrascosa,1985; Galili,1995).

Por otra parte, están apareciendo cada vez más trabajos de investigación que relacionan la historia y epistemología de la ciencia con la enseñanza (Wandersee, 1992). Muchos de estos trabajos (Matthews,1990; Burbules y Lin, 1991) sugieren que algunas dificultades en el aprendizaje de los conceptos científícos pueden tener cierto paralelismo con los problemas que se presentaron en la formación de los mismos a lo largo de la historia, si bien se han de aceptar las limitaciones derivadas de las diferencias de contexto - de indagación y culturales-en los que surgen (Saltiel y Viennot, 1985). Así pues, el análisis histórico y epistemológico de los problemas 
habidos en Ia formación de conceptos y teorías científicas puede servir de ayuda para clarificar las posibles causas de estas dificultades de aprendizaje (Giannetto et al., 1992).

De acuerdo con lo anterior, el problema investigado es doble. Por un lado, analizaremos las dificultades de aprendizaje que tienen los estudiantes de secundaria y universidad en las teorías y conceptos básicos empleados actualmente en la interpretación de los fenómenos electrostáticos y, por otro, analizaremos en qué medida existe un cierto paralelismo entre estas dificultades y los problemas epistemológicos que hubo de superar la historia de la electricidad hasta constituirse como ciencia. En particular, dentro de la electricidad el marco teórico conceptual que se analiza en este artículo abarca desde eI concepto de carga eléctrica hasta la teoría elemental de campo eléctrico, que se puede resumir en el cuadro I. Este marco teórico, exceptuando el concepto de potencial eléctrico, es el considerado en los contenidos de electrostática para el nuevo bachillerato (MEC, 1991).

Empezaremos a plantear el problema analizando cuáles han sido los principales saltos epistemológicos en la construcción de la electricidad. En particular, el conocimiento de esta ecología conceptual en un dominio, o teoría específica (Touimin,1972), puede ser una herramienta interesante para aquel profesor que quiera plantearse la práctica docente como proceso de construcción de conocimientos, donde necesariamente se han de tener en cuenta las ideas y habilidades iniciales de los aprendices.

\section{¿QUÉ PROBLEMAS EPISTEMOLÓGICOS IMPORTANTES SE PRESENTARON EN LA CONSTRUCCIÓN DE LA ELECTRICIDAD?}

A modo de síntesis epistemológica general (Heilbron, 1979; Whittaker, 1987; Taton, 1985), se puede afirmar que los principales problemas cuya solución supuso un avance significativo en la construcción de la electricidad como ciencia fueron los siguientes:

a) La búsqueda de explicación de los fenómenos triboeléctricos (electrización de los cuerpos por frotamiento) condujo a la hipótesis general de que la materia es

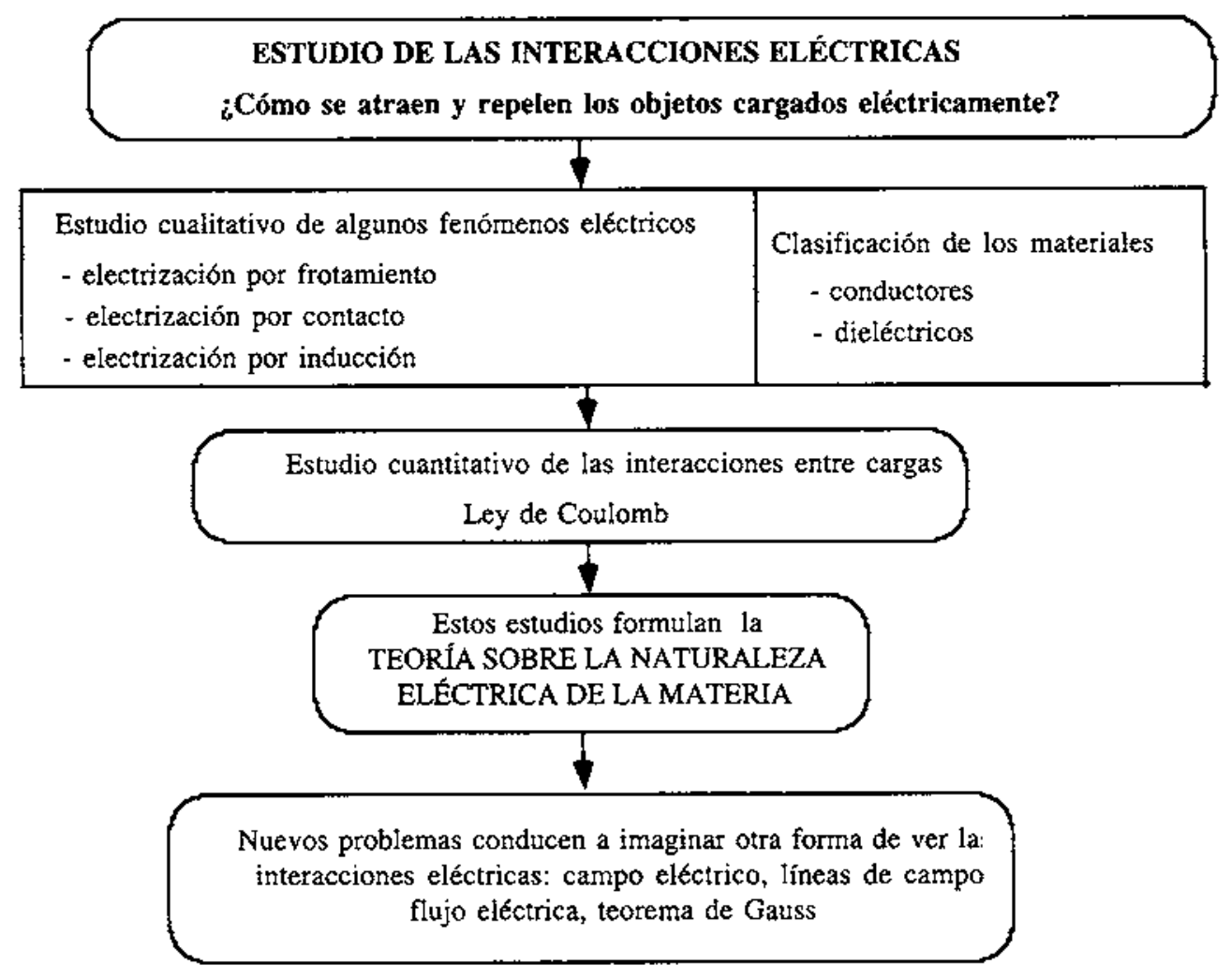


Cuadro II

Conocimientos conceptuales y procedimentales necesarios para interpretar el fenómeno.

\section{Fenómeno considerado}

1. Electrización por frotamiento de un plástico con un paño de lana.

2. No electrización por frotamiento de un metal con un paño de lana.

3. Electrización por contacto y repulsión entre cuerpos cargados de la misma manera.

4. Electrización de un objeto ligero por influencia o inducción de otro cargado.

5. Electrización por inducción đe un cuerpo extenso (dieléctrico) por otro cargado, o transmisión de la epropiedad eléctrica" a través de un medio. También llamado polarización del dieléctrico.

\section{Conocimientos y habilidades para explicarlo}

1. Se supone que la materia es electrica, es decir, tiene cargas positivas y negativas antes de frotar. A partir de aquí. Ja electrización se explica por intercambio de cargas entre los cuerpos frotados.

2. Se sigue aceptando coherentemente que el metal también es eléctrico, pero se introduce como nueva hipotesis que el metal conduce (es decir, facilita el paso de cargas) y, por tanto, se descarga si no está aislado.

3. Se supone que el cuerpo cargado transporto parte de su carga al descargado y se explica la repulsion por el principio emptrico de que cargas del mismo signo se repelen.

4. El conocimiento explicativo requerido es más complejo. La secuencia argumental a utilizar podría tener las siguientes etapas: a) Se acepta que el objeto neutro tiene cargas positivas y negativas.

b) Se supone que, al acercar el cuerpo cargado al objeto jigero, se produce en éste una separación de las cargas, según el principio empírico anterior.

c) La mayor proximidad de cargas de distinto signo justifica que la fuerza atractiva sea superior a la repulsiva y, por tanto, el cuerpo cargado atrae al ligero (uso cualitativo de la ley de Coulomb).

5. Son posibles varios argumentos correctos de distinto nivel conceptual :

a) Mediante interpretación coulombiana semejante al anterior fenómeno, pero transmitiendo la perturbación a través de las partículas del dieléctrico.

b) Mediante la introducción del campo eléctrico creado por el cuerpo cargado y transmitido a través del medio. eléctrica (es decir, posee cargas) aunque aparentemente se presente como neutra (igual cantidad de cargas positivas y negativas). La representación mental de la «propiedad eléctrica» (que manifiestan los cuerpos frotados) como una especie de «halo» (según Gilbert, 1600) fue superada por otra imagen «sustancialista», donde a Ia carga se le consideraba un (dos) fluido(s) especial(es) que poseían todos los cuerpos (modelo hidrostático de carga según Franklin, 1747).

b) La profundización en el estudio cuantitativo de las interacciones que se ejercen mutuamente las cargas eléctricas y su fundamentación analógica en la mecánica newtoniana permitió consolidar aquella hipótesis general y definir operativamente el concepto de carga eléctrica (Coulomb, 1785).

c) Finalmente, el problema de la transmisión de la interacción eléctrica a través de un medio, junto al de la unificación de las diferentes electricidades y del magnetismo, mostraron las insuficiencias del modelo hidrostático de la carga y permitieron un salto cualitativo impor- tante que condujo a la introducción de la teoría del campo de fuerzas electromagnéticas (Faraday, 1846) y, posteriormente, a la síntesis representada por la ecuaciones de Maxwell (1864).

Este último programa de investigación, que unificó las explicaciones de las diferentes interacciones eléctricas y magnéticas conocidas (Berkson, 1981), supuso un cambio ontológico radical respecto a la manera coulombiana de concebir la carga y la interacción eléctrica. En efecto, este cambio que se inicia con Faraday logra complementar la visión cosmológica newtoniana (donde la materia $y$ el espacio se consideran entidades separadas, absolu. tas e independientes), que había servido de marco filosó. fico en la definición coulombiana de interacción eléctrica, con la visión cosmológica de tradición cartesiana donde la materia y el espacio se presentan como insepa* rables. El esfuerzo de imaginación puesto de manifiesto al visualizar la interacción eléctrica en el medio (apoyada en la incipiente filosofía kantiana de fines del XVIII, superadora del empirismo y del racionalismo) facilitó la construcción de una teoría que pudiera explicar de ma- 
nera unitaria las interacciones electromagnéticas (Thuillier, 1989), teoría que se concretó en la introducción del concepto director đe campo de fuerzas.

A partir de este marco general de problemas epistemologicos que tuvo que resolver la comunidad científica, no es difícil emitir hipótesis razonables sobre las posibles dificultades que se pueden presentar en el aula. Por otra parte, el conocimiento de la epistemología de la ciencia se reconoce por los investigadores como un heurístico valioso para los profesores a la hora de seleccionar y secuenciar el currículo (Wandersee et al., 1994). En nuestro caso ayudará a secuenciar el estudio de los fenómenos triboeléctricos como situaciones problemáticas de interés, cuyo tratamiento cientéfico permitirá introducir los conceptos básicos, y comprender la naturaleza eléctrica de la materia (Gil et al., 1991).

\section{¿QUÉ CONOCIMIENTOS Y HABILIDADES COGNITIVAS DEBERIAN TENER LOS ESTUDIANTES PARA INTERPRETAR LOS FENOMENOS TRIBOELECTRICOS Y QUE DIFICULTADES SE LES PUEDEN PRESENTAR?}

Aunque no podemos detenernos ni siquiera en una mínima descripción de lo que podría ser un microcurrículo de electrostática en la enseñanza del bachillerato (16-18 años), tendremos que indicar cuáles pueden ser los conocimientos y habilidades cognitivas que han de poseer y articular los estudiantes para poder interpretar científicamente los fenómenos electrostáticos que se presentan, de ordinario, en el aula. A partir de estos objetivos conceptuales y procedimentales «terminales» que han de lograr los estudiantes al finalizar el estudio del tema, y de las concepciones y razonamientos que tenían inicialmente, podremos avanzar hipótesis sobre las dificultades de aprendizaje que se pueden presentar.

De entrada, se puede suponer que los conocimientos conceptuales y estratégicos contenidos en el cuadro II, constituyen las metas instructivas perseguidas, de acuerdo con su clasificación en objetivos conceptuales, procedimentales y actitudinales (MEC, 1991). Estos objetivos pueden lograrse, por ejemplo, al proponer el estudio de los fenómenos en un programa de investigación dirigida (Furi6, 1994; Duschl, 1995).

En cuanto a las dificultades de aprendizaje que se pueden presentar, se puede recurrir a los resultados de la investigación sobre concepciones alternativas y a su posible influencia, sobre todo si la enseñanza no es eficaz. La abundante investigación en este campo ha mostrado que una de las características de algunas preconcepciones es su similitud con concepciones vigentes a lo largo de la historia del pensamiento científico y filosófico (Nussbaum, 1983). Un ejemplo de paralelismo entre ambos tipos de ideas que hemos estudiado es el existente entre las ideas que sobre la naturaleza material de los gases se tenía en la química precientífica y las concepciones actuales de los alumnos (Furió et al., 1987). En efecto, el carácter casi inmaterial que la gente atribuye a los gases, es consistente con el que le atribuían los químicos escolásticos antes del nacimiento de la química como ciencia moderna. No en balde hubo en la historia de la «filosofía natural» cuatro siglos dedicados al estudio de la «química neumática», que concluyó en el momento que los gases se consideraron «tan corpóreos» como los sólidos y los líquidos. En hipótesis, un paralelismo análogo se puede vaticinar entre las ideas actuales de los estudiantes sobre la naturaleza eléctrica de la materia y los modelos preteóricos acerca de la «propiedad eléctrica y su transmisión», que tanto contribuyeron a la construcción de la electricidad. En consecuencia, no parece descabellado plantear una primera hipótesis que afirme:

H.1. Las interpretaciones de los estudiantes a cuestiones sobre la causalidad de los fenómenos de electrostatica presentarán - a pesar de la instrucción-mayoritariamente ideas sobre la naturaleza eléctrica de la materia, basada en un modelo hidrostático similar al propuesto por Franklin.

La existencia de estas ideas cualitativas sobre el concepto de carga permitirá la solución de algunos de los problemas planteados, pero también provocará la aparición de dificultades epistemológicas al igual que sucedió históricamente (Apartado 2). Por ejemplo, si el estudiante acepta la existencia de carga en la materia y tiene una representación hidrostática de la carga, será fácil que explique el fenómeno de electrización por contacto (Párrafo 3 del cuadro I), pero tendrá dificultades para explicar lainducción electrostática en un cuerpo ligero (Párrafo 4), y su modelo será claramente insuficiente para interpretar este mismo fenómeno en un dieléctrico extenso (Párrafo 5). Es decir, relacionada con la hipótesis anterior se puede explicitar una segunda hipótesis:

H.2. Existe cierto paralelismo entre los problemas históricos del modelo hidrostático de carga fe incluso el de la teoría mecanicista de Coulomb), a la hora de explicar las interacciones entre cargas y los principales obstáculos encontrados en el aprendizaje de la electrostática.

Por otra parte, y coherentemente con esta segunda hipótesis, también cabe esperar que estas dificultades vayan gradualmente en aumento conforme se vayan planteando sucesivamente la secuencia de fenómenos del cuadro II. Esta gradación de dificultad epistemológica debe llegar a un máximo cuando la explicación del fenómeno requiera conocimientos como el del campo eléctrico, cuya significación física se supone que tiene un estatus muy bajo entre la población estudiantil (Bachelard, 1940; Mortimer,1995). En definitiva, una tercera hipótesis relacionada con el paralelismo entre los problemas históricos y las dificultades de aprendizaje es :

H.3. El aprendizaje significativo del concepto científico de campoeléctrico logrado en estudiantes, incluso universitarios, que han recibido enseñanzas convencionales de electricidad será muy pobre. En consecuencia, su utilización en la explicación de los fenómenos electrostáticos será muy minoritaria. 
Para efectuar la contrastación de esta última hipótesis, podemos considerar que un estudiante ha aprendido significativamente la ídea de campo eléctrico cuando resuelve correctamente las siguientes situaciones :

a) Diferencia claramente entre fuerza y campo eléctrico.

b) Reconoce la importancia del medio en la transmisión de la interacción eléctrica (es decir, sabe que no se trata de una acción a distancia independiente del medio, ni es instantánea).

c) Se discrimina claramente entre una aplicación conlombiana y una de campo en cuestiones de conflicto cognitivo tipo «jaula de Faraday».

\section{DISEÑO EXPERIMENTAL}

Se ha ideado un diseño longitudinal y variado para analizar si persisten dificultades de aprendizaje en estos conceptos de carga y campo eléctrico a lo largo de la enseñanza secundaria y universitaria.

En el diseño elaborado se han incluido, como instrumentos, un cuestionario con ocho preguntas de tipo abierto con énfasis en las explicaciones y una entrevista estructurada, a una muestra de estudiantes de los diferentes cursos. Este segundo instrumento trata de observar si se contrastan, de forma cualitativa y convergente, los análisis de los resultados obtenidos en Ios cuestionarios.

\section{Características de las muestras de estudiantes que han cumplimentado el cuestionario}

El cuestionario se ha pasado a un total de 268 alumnos distribuidos en cinco grupos a los que designaremos como:

G0. Alumnos de $2^{\circ}$ de BUP (15-16 años) que estudian por primera vez un curso de física y química en secundaria (aún no iniciados en el estudio formal de la electricidad) $y$, por tanto, puede considerarse como nivel inicial de referencia. El currículo de electrostática estudiado por estos estudiantes se refiere a los fenómenos triboeléctricos y al establecimiento de la carga eléctrica como propiedad general de la materia. Así pues, sólo cumplimentaron la primera parte del cuestionario referido al concepto de carga eléctrica y, obviamente, no se les pidieron las preguntas relativas al campo eléctrico. El número de estudiantes de este grupo fue de 31.

G1. Alumnos de $3^{\circ}$ de BUP (16-17 años) de dos centros privados de San Sebastián, que han recibido instruccion sobre la naturaleza eléctrica de la materia, así como su aplicación a la interpretación de fenómenos eléctricos elementales. Asimismo, en el currículo se ha estudiado el concepto de campo eléctrico creado por una o varias cargas puntuales. El número de estudiantes de este grupo fue de 61 .
G2. Alumnos de COU (17-I8 años) de dos centros privados de San Sebastián que, además de los contenidos del grupo anterior, han recibido instrucción sobre el concepto de campo eléctrico creado por un cuerpo extenso cargado y han estudiado el teorema de Gauss y sus aplicaciones. El número de estudiantes de este grupo fue de 60 .

G3. Alumnos de primer curso de la Facultad de Ciencias Físicas de la Universidad del País Vasco, cuyo currículo incluye el cálculo del campo eléctrico para distribuciones continuas de carga en conductores y dieléctricos. El número de estudiantes de este grupo fue de 60 .

G4. Alumnos de tercer curso de la Facultad de Ciencias Físicas de la Universidad del País Vasco que, además de los contenidos anteriores, han recibido instrucción sobre las leyes de Maxwell de la electrodinámica clásica. EI número đe estudiantes de este grupo fue de 64 .

El cuestionario fue aplicado por el mismo investigador a todos los grupos cuando los estudiantes acababan de terminar los temas de electrostática y corriente continua correspondientes a sus respectivos programas. En el caso de los estudiantes del grupo G4, aunque ya habían dado los contenidos necesarios para el cuestionario, se realizó la encuesta después de dar el primer cuatrimestre de la asignatura sobre electromagnetismo. Los alumnos cumplimentaron el cuestionario en concepto de examen (sin poderse comunicar entre ellos), durante una clase cuya duración fue de 55 minutos. Es de reseñar que, en general, los estudiantes (incluso los de los niveles más bajos) no tuvieron ninguna dificultad de comprensión respecto al enunciado de las preguntas. El cuestionario fue validado por un grupo de seis profesores expertos que estuvieron de acuerco al cien por cien en los objetivos perseguidos en cada ítem.

\section{Contenido del cuestionario aplicado y de las entrevistas realizadas}

Los criterios que nos han guiado al elegir las situaciones físicas presentadas en las cuestiones se han basado en los objetivos de aprendizaje especificados en el apartado 3. Así, el cuestionario consta de dos partes, la primera parte contiene cinco preguntas para analizar como interpretan los diferentes fenómenos electrostáticos expuestos en el apartado $3 ; y$ la segunda, tres cuestiones para ver si han aprendido significativamente el conceptode campoeléctrico (Anexo).

El segundo instrumento para contrastar las hipotesis emitidas ha consistido en entrevistar sobre varios fenómenos triboeléctricos y el fenómeno de la «jaula de Faraday» a 24 estudiantes. Las entrevistas tienen como objetivo: a) ver cómo explican los estudiantes dos experiencias de triboelectrización sencillas (Ferreira, 1987); b) contrastar si estas explicaciones se aproximan o no a la teoría de la naturaleza eléctrica de la materia actualmente aceptada; y c) ver si aplican de forma significativa el concepto de campo eléctrico. Las entrevistas han tenido una duración media de 30 minutos y han sido 
Tabla I

Porcentajes de respuestas obtenidos en las explicaciones de la electrización de una barta de plástico.

\begin{tabular}{|c|c|c|c|c|c|}
\hline \multirow[b]{2}{*}{$\begin{array}{l}\text { Categoria de } \\
\text { respuesta para } \\
\text { el ítem 1 }\end{array}$} & \multicolumn{5}{|c|}{ Porcentaje de respuesta (\%) } \\
\hline & $\begin{array}{c}2^{\circ} \text { de BUP } \\
N=31\end{array}$ & $\begin{array}{c}3^{\circ} \text { de BUP } \\
N=61\end{array}$ & $\begin{array}{l}\mathrm{COU} \\
\mathrm{N}=60\end{array}$ & $\begin{array}{c}1^{\circ} \text { de Ffsicas } \\
\mathrm{N}=60\end{array}$ & $\begin{array}{c}3^{\circ} \text { de Ffsicas } \\
N=64\end{array}$ \\
\hline «Eléctricos» $(A)^{*}$ & 49 & 60 & 66 & 77 & 77 \\
\hline $\begin{array}{l}\text { «Intercambio de } \\
\text { cargas» (A.I) }\end{array}$ & 22 & 36 & 42 & 52 & 57 \\
\hline $\begin{array}{l}\text { «Separación de } \\
\text { cargas" (A.2) }\end{array}$ & 27 & 24 & 24 & 25 & 20 \\
\hline «Creacionistas" (B) & 20 & 10 & 13 & 6 & 8 \\
\hline Incodificables & 20 & 18 & - & 8 & - \\
\hline No contesta & 11 & 12 & 21 & 10 & 15 \\
\hline
\end{tabular}

* Respuesta correcta

grabadas en audio para su posterior transcripción y análisis. La estructura de la entrevista consta de tres partes. En primer lugar, se pregunta al entrevistado antes de realizar la experiencia qué piensa que sucederá, estimulándole a que emita una hipótesis lo más fundamentada posible. En segundo lugar, se realiza la experiencia y el entrevistado observa lo que sucede. Por último, el entrevistado explica lo ocurrido confirmando la hipótesis emitida o bien trata de dar una nueva explicación.

\section{RESULTADOS OBTENIDOS Y DISCUSIÓN}

Para facilitar la presentación y discusión de los resultados obtenidos, se expondrán en diferentes apartados y se incluirán resultados cualitativos extraídos de las entrevistas que ilustran las interpretaciones realizadas.

\section{Explicaciones de los estudiantes sobre algunos fenómenos triboeléctricos}

Respecto de la existencia de cargas en la materia, las respuestas de los estudiantes al ítem I se pueden clasificar en dos categorías (Tabla I): la categoría A, denominada "eléctricos", que acepta la existencia de cargas en la materia, y la categoría $B$, denominada «creacionistas», que explica que la carga se crea en el momento en que se frota o se hace trabajo sobre el cuerpo. Así pues, los estudiantes asumen mayoritariamente (entre un 50\% y un $77 \%$ ) que la materia antes de frotar es eléctrica y, aunque el porcentaje de respuestas incorrectas es pequeño, se mantiene a lo largo de la instrucción y persiste en los cursos universitarios $\left(6 \%\right.$ en $1^{\circ}$ y $8 \%$ en $3^{\circ}$ ). Dentro de la categoría A («eléctricos») se detectan dos tipos de explicaciones (Tabla 1). La primera de ellas (A.1, correcta) indica que se produce un intercambio de cargas entre los cuerpos frotados. En la segunda (A.2) se explica que la electrización es debida a que en la barra de plástico se produce una separación local de las cargas positivas y negativas. En nuestra opinión, la posible causa del error cometido en esta explicación puede estar en que los alumnos recuerdan que los cuerpos tienen cargas positivas y negativas distribuidas aleatoriamente, y que por induccion se separan localmente (sin que el cuerpo esté cargado externamente). Es preocupante que esta explicación se mantenga a lo largo de la instrucción en torno al $25 \%$ del total de las respuestas.

En cuanto a las explicaciones de los estudiantes respecto a la no electrización por frotamiento de una barra metálica (ítem 2), se pueden clasificar en dos grandes categorías (Tabla II). En la categoría A, los estudiantes argumentan de forma correcta que, aunque se produce un 
Tabla II

Porcentaje de respuestas obtenidas al explicar la no-electrización de una barra metálica frotada.

\begin{tabular}{|c|c|c|c|c|c|}
\hline \multirow[b]{2}{*}{$\begin{array}{l}\text { Categoría de } \\
\text { respuesta para } \\
\text { el ítem } 2\end{array}$} & \multicolumn{5}{|c|}{ Porcentaje de respuesta (\%) } \\
\hline & $\begin{array}{c}2^{\circ} \text { de BUP } \\
N=31\end{array}$ & $\begin{array}{c}3^{\circ} \text { de BUP } \\
N=6 !\end{array}$ & $\begin{array}{l}\mathrm{COU} \\
\mathrm{N}=60\end{array}$ & $\begin{array}{c}1^{\circ} \text { de Físicas } \\
N=60\end{array}$ & $\begin{array}{c}3^{\circ} \text { de Físicas } \\
N=64\end{array}$ \\
\hline $\begin{array}{l}\text { "Trasvase de cargas" } \\
\text { (A) }{ }^{*}\end{array}$ & 12 & 23 & 23 & 23 & 21 \\
\hline $\begin{array}{l}\text { «No hay trasvase } \\
\text { de cargas»(B) }\end{array}$ & 64 & 47 & 49 & 56 & 47 \\
\hline «Debido al metal»(B.1) & 39 & 31 & 43 & 38 & 44 \\
\hline «Debido al paño» (B.2) & 9 & - & 6 & 12 & - \\
\hline «Frotar más» (B.3) & 16 & 16 & - & 6 & 3 \\
\hline Incodificables & 20 & 19 & 15 & 11 & 11 \\
\hline No contesta & 4 & 11 & 13 & 11 & 21 \\
\hline
\end{tabular}

*Respuesta correcta

trasvase o una separación de cargas, al ser el metal un buen conductor las cargas, se desplazan a través de la barra sin que ésta se electrice. En la corrección de las respuestas se han considerado también correctas aquéllas que razonando como se ha indicado hablaban de «separación de cargas». Sin embargo, una mayoría no tiene en cuenta el carácter conductor de la barra y argumenta (categoría B) que no se produce el trasvase o separación de cargas por diferentes razones, como las características de la barra que impedirían su electrización (categoría B.1, mayoritaria), las características del paño con que se frota (categoría B.2) o bien porque no se frota lo suficiente (categoría B.3). Esta última respuesta sería convergente con las explicaciones que hemos llamado en el ítem I «creacionistas» y su porcentaje sería, por ello, similar al que aparecía entonces. Es decir, la coherencia de las respuestas dadas por Ios estudiantes a los ítems 1 y 2 justifican estos resultados.

Las dificultades de los estudiantes en el ítem 2 aumentan respecto a las dificultades del anterior, ya que para realizarlo se necesitan argumentos que se articulan en dos etapas: a) admitir el intercambio o separación de cargas; b) tener en cuenta que, al no estar la barra de metal aislada (aunque esta condición no se indica a proposito en el enunciado), se produce una conducción de cargas. Los bajos resultados obtenidos (alrededor de un $22 \%$ en estudiantes universitarios) pueden explicarse por la utilización de un causalismo simple (dificultad metodológica), característico de la metodología de sentido común (Gil y Carrascosa, 1985), donde se buscan argumentos directos y rápidos que expliquen el fenómeno. Es decir, la forma más sencilla de aplicar la teoría para resolver una cuestión consiste en argumentar con rapidez, usando razonamientos que tengan una única causa que la resuelva puntualmente. La búsqueda de coherencia puntual es típica del pensamiento ordinario a diferencia del pensamiento científico, más riguroso, cuyos razonamientos buscan la validación de las respuestas viendo si son coherentes globalmente con el cuerpo teórico (Furió, 1996).

Ei análisis de las entrevistas estructuradas es convergente con las categorías establecidas en los cuestionarios como se puede comprobar en los ejemplos siguientes:

\section{Ejemplo de explicación clasificada en la categoría} "creacionista" (Nuria, $2^{\circ}$ de BUP)

Entrevistador (E): ¿Cómo crees que se ha cargado el cuerpo frotado?

Alumna (A): Bueno, antes no hay cargas, pero como el rozamiento crea calor... pues debido al calor aparecen cargas en la pajita. Pero no sé de que signo serán. 
E: Antes de frotar la pajita de plástico, ¿tenía cargas?

A: No, antes el cuerpo está descargado; es debido al rozamiento que tiene cargas.

\section{Ejemplo de explicación clasificada en la categoría de} "eléctricos» (Alberto, $3^{\circ}$ de BUP)

Entrevistador: Al frotar la pajita, ¿cómo se ha cargado?

Alumno: Antes de frotar tiene el mismo número de cargas positivas y negativas, pero después se descompensan debido al frotamiento. Es decir, la pajita queda cargada.

Más explícito es otro de los estudiantes del mismo nivel cuando argumenta:

Alumno: Si no está cargada la pajita, es que ni sobran ni faltan cargas positivas y negativas. Al frotar, perdería electrones y se queda cargada.

3. Otro ejemplo de explicación clasificada en la categoría de "eléctricos» (Gorka, $1^{o}$ de Físicas)

Entrevistador: ¿Qué sucede cuando frotamos la pajita con la servilleta de papei?

Alumno: Al frotar la pajita contra el papel saltan cargas. Bueno, yo no sé decir si se carga positivamente o nega. tivamente; yo creo que negativamente.

E: ¿A qué es debido este paso de cargas?

A: Debido al rozamiento, la pajita capta electrones del papel y se carga negativamente.

Otra respuesta más elaborada desde el punto de vista teórico es la de esta otra alumna (Nekane, $1^{\circ}$ de Físicas):

Alumna: Después de frotar en la pajita, el número de cargas positivas y negativas no es el mismo. Entonces hay una carga neta en ella que puede ser o negativa o positiva. Esto es debido a que ha habido una transferen. cia de cargas entre el papel y la pajita.

4. Ejemplo de explicación clasificada en la categoría "no hay trasvase de cargas" (B.3 del item 2. Alfonso, COU):

Entrevistador: ¿A qué es debido que la barra de metal no se electrice al frotarla?

Alumno: Bueno, yo creo que sí se carga pero hay que frotar más, ya que la barra de plástico y la barra de metal son materiales diferentes. En el caso de la barra de plástico enseguida se calienta, mientras que a la de metal le cuesta más.

E: ¿Antes de frotar hay cargas en la barra de metal?

A: No, aparecen al frotarla.

E: Ahora la vamos a frotar durante un buen rato y después la acercamos al péndulo electrostático. (Se realiza el experimento). Puedes comprobar que sigue sin cargarse.
A: Bueno, no sé. Yo creo que se debería cargar... No sé.

La gran mayoría de los estudiantes (alrededor de un $80 \%$ ) explica de forma correcta el fenómeno presentado en el ítem 3. Esto puede ser debido a que la respuesta correcta puede ser fácilmente explicada basándose en $u n$ modelo hidrostático de carga, donde, al pasar carga de la barra a la bolita, ésta queda cargada con el mismo signo y, por tanto, se repelen.

\section{Dificultades en la interpretación de fenómenos de polarización como síntoma de la persistencia en los estudiantes del modelo hidrostático de carga}

Cuando los estudiantes tratan de analizar fenómenos de inducción o de influencia local (ítem 4), se constata cómo vuelve a elevarse el nivel de dificultad de la pregunta, pues solamente contestan correctamente el $36 \%$ en el grupo de $3^{\circ}$ de Físicas. También se constata una diversificación de los argumentos, algunos de los cuales recuerdan interpretaciones anteriores a las del modelo coulombiano, como se puede ver en la tabla III. En efecto, la categoria de respuesta $A$ que explica correctamente este fenómeno se ha subdividido en dos, según se haga basándose en el modelo coulombiano (A.I) o en la idea de campo (A.2). Puede comprobarse cómo este último tipo de explicaciones es minoritario, incluso, en el nivel más elevado (un $5 \%$ en $3^{\circ}$ de Físicas). La categoría $B$ es una primera concepción alternativa similar al modelo de Gilbert, donde se habla del efecto «halo» producido por los cuerpos cargados para explicar la atracción que ejercen sobre cuerpos ligeros situados en su proximidad. La categoría $C$ representa un segundo modelo alternativo similar al modelo de Franklin, donde se justi. fica la atracción de los cuerpos cargados sobre la base de la existencia de dos tipos de cargas de signo opuesto. Es de destacar que el porcentaje de respuestas no contestadas aumenta a medida que sube el nivel del curso.

La explicación correcta del fenómeno de acuerdo con el modelo coulombiano va aumentando lentamente con la instrucción, aunque una de las explicaciónes (utilización del modelo de campo eléctrico) obtiene porcentajes muy pequeños. En nuestra opinión, el porcentaje de respuestas correctas es muy bajo (sobre el $20 \%$ en secundaria y el $30 \%$ en estudiantes universitarios de ciencias físicas) teniendo en cuenta que el fenómeno de inducción está contemplado en todos los programas de electrostática desde secundaria. Así mismo, queremos subrayar que la incidencia de la instrucción es mínima, siendo particularmente reseñable la baja utilización del modelo de campo eléctrico en alumnos con varios años de instrucción en la materia.

\section{Ejemplo de respuesta calificada como similar al modelo de Franklin (Joseba, $I^{\circ}$ de Físicas)}

Alumno: La atracción siempre se produce por cargas de diferente signo pero, como me has indicado, aquí el trocito de aluminio no tiene carga. Francamente, no sé... no sé... lo he experimentado miles de veces; además es la típica explicación que te dan siempre. 
Tabla III

Porcentaje de respuestas obtenidas al explicar la atracción de trocitos de papel por un cuerpo cargado.

\begin{tabular}{|c|c|c|c|c|c|}
\hline \multirow[b]{2}{*}{$\begin{array}{l}\text { Categoría } \\
\text { de respuesta } \\
\text { para el f́tem } 4\end{array}$} & \multicolumn{5}{|c|}{ Porcentaje de respuesta (\%) } \\
\hline & $\begin{array}{c}2^{\circ} \text { de BUP } \\
\mathrm{N}=31\end{array}$ & $\begin{array}{c}3^{\circ} \text { de BuP } \\
\mathrm{N}=61\end{array}$ & $\begin{array}{l}\mathrm{COU} \\
\mathrm{N}=60\end{array}$ & $\begin{array}{c}I^{\circ} \text { de Fisicas } \\
N=60\end{array}$ & $\begin{array}{c}3^{\circ} \mathrm{de} \mathrm{Fisicas} \\
\mathrm{N}=64\end{array}$ \\
\hline "Coulomb» (A.1)* & - & 13 & 27 & 30 & 31 \\
\hline «Campo" $(\mathrm{A} 2)^{*}$ & - & - & - & 3 & 5 \\
\hline «Gilbert» (B) & 50 & 38 & 38 & 23 & 17 \\
\hline «Franklin» (C) & 34 & 29 & 22 & 25 & 19 \\
\hline Incodificables & 10 & 13 & - & - & - \\
\hline No contesta & 6 & 7 & 13 & 19 & 28 \\
\hline
\end{tabular}

* Respuesta correcta

Entrevistador: ¿Y qué explicación te dan?

A: El que uno tiene carga de un signo y el otro de distinto, y se atraen.

E: Pero ya ves que el trocito de aluminio está sin carga.

A: Sí. Tal vez sea que el papel de aluminio se carga de alguna forma, y luego se produce la atracción.

2. Ejemplo de respuesta calificada como similar al modelo de Gilbert (Alberto, $3^{\circ}$ de BUP)

Entrevistador: ¿Por qué atrae la pajita al trocito de altuminio?

Alumno: La pajita está cargada, ya no tiene el mismo número de electrones que de protones. El trocito de aluminio detector no está cargado. Entonces la pajita atraerá a cuerpos metálicos pequeños.

E: Si utilizásemos un trocito de papel también pasaría lo mismo. Recuerda el caso del bolígrafo frotado y los pedacitos de papel que habéis hecho en clase.

A: La pajita atraerá a cualquier cuerpo pequeño de alrededor, aunque a unos más que a otros.

3. Ejemplo de respuesta correcta después de reflexionar sobre la respuesta inicial (Iñaki, COU)
Entrevistador: ¿Por qué le atrae la pajita?

Alumno: Porque la carga del aluminio del detector es diferente a la de la pajita, y cargas de distinto signo se atraen.

E: Pero la pajita está cargada y el trocito de aluminio no. ¿Se atrae una cosa cargada con una neutra?

A: ¡Ah!, bueno. Esto está neutro, ¿no? Pero se pueden desplazar las cargas o, como si dijéramos, las cargas positivas se podrían poner a un lado y las cargas negativas al otro, ¿no? Entonces, la pajita que está cargada positivamente atrae a la parte negativa.

\section{Ejemplo de respuesta correcta (June, $3^{\circ}$ de Físicas)}

Entrevistador: ¿Por qué le atrae la pajita?

Alumna: Se produce una inducción de la pajita al trocito de alumnio del detector.

E: ¿Podrías explicar en qué consiste la inducción?

E: Pues, el trocito de aluminio está neutro; es decir, tiene el mismo número de cargas positivas que de negativas. Pero, por la influencia de la pajita cargada, las cargas de un signo están en un lado y las de otro signo en otro. Por tanto, unas están más cerca y, al estar las de distinto signo más próximas, las fuerzas son mayores, entonces se produce la atracción. 
Tabla IV

Porcentajes de respuesta obtenidos al explicar la atracción eléctrica de una bolita, debido a la inducción ejercida por vna barra de madera polarizada.

\begin{tabular}{|l|c|c|c|c|}
\hline & \multicolumn{4}{|c|}{ Porcentaje de respuesta (\%) } \\
\hline $\begin{array}{l}\text { Categoría } \\
\text { de respuesta } \\
\text { del ítem S }\end{array}$ & $\begin{array}{c}3^{\circ} \text { de BUP } \\
\mathrm{N}=6 !\end{array}$ & $\begin{array}{c}\text { COU } \\
\mathrm{N}=60\end{array}$ & $\begin{array}{c}1^{\circ} \text { de Físicas } \\
\mathrm{N}=60\end{array}$ & $\begin{array}{c}3^{\circ} \text { de Físicas } \\
\mathrm{N}=64\end{array}$ \\
\hline $\begin{array}{l}\text { Existe atracción (A)* } \\
\text { Modelo coulombiano (A.1) }\end{array}$ & 10 & 4 & 27 & 20 \\
Modelo de campo (A.2) & 8 & 2 & 19 & 14 \\
\hline No se ejerce influencia (B) & 2 & 2 & 8 & 6 \\
\hline uLa madera no conduce» (B.1) & 67 & 90 & 52 & 66 \\
\hline No lo sé & 16 & 70 & 44 & 53 \\
\hline
\end{tabular}

* Respuesta correcta

En la interpretación del fenómeno de inducción presentado en el ítem 5 es necesario tener en cuenta la polari. zación del medio (la barra de madera). Esto aumenta el nivel de dificultad respecto del fenómeno de inducción que se ha analizado en el ítem anterior y, por tanto, el porcentaje de respuestas correctas es mucho más bajo (Tabla IV).

La categoría $A$ de respuesta se basa en un modelo coulombiano (inducción eléctrica y transmisión de su efecto) o en un modelo de campo (la acción eléctrica se propaga polarizando el dieléctrico constituido por la barra de madera). La categoría $B$ se basa en un pensamiento espontáneo del alumno, que consiste en indicar que solamente es posible la atracción si la carga se puede transmitir por la barra de madera y, al ser ésta aislante, no será posible atraer a la bolita. Este tipo de análisis es similar al que realiza el modelo de Franklin, donde la interacción eléctrica se realiza a través del llamado «fluido eléctrico». Esta respuesta es ampliamente mayoritaria, manteniéndose a lo largo de los cursos.

Aunque hay un salto cualitativo de un $15 \%$ en el porcentaje de respuestas correctas entre la enseñanza media y universitaria, es evidente que en el nivel universitario sólo una cuarta parte de los estudiantes interpreta correc. tamente el fenómeno a pesar de su familiarización con el mismo. En nuestra opinion, muchas de las dificultades de la mayoría de los estudiantes no son solamente de tipo conceptual sino también procedimental o estratégico. En este caso particular, la fijación funcional de una repre- sentacion mental aprendida como la de la clasificación de los materiales en conductores y aislantes es la que impide ver formas alternativas de explicación. Ésta es una de las dificultades mencionadas por la psicología cognitiva en la resolución ordinaria de problemas (Anderson, 1990).

\section{¿En qué medida utilizan los estudiantes de forma significativa el concepto de campo eléctrico?}

De acuerdo con la segunda consecuencia de la hipótesis, se supone que los estudiantes no diferenciarán fácilmente entre los conceptos de intensidad de campo y de fuerza eléctrica, incluso en situaciones familiares dentro de un contexto escolar (item 6). De la misma forma, cuando se presenten situaciones de conflicto cognitivo que requieran una aplicación significativa de la idea del campo eléctrico, se elevará el grado de dificultad de los estudiantes (items 7 y 8 ).

En el ítem 6 se trata de detectar si existe confusión entre los conceptos de fuerza eléctrica y campo eléctrico en una cuestión de tipo operativo, formulada dentro de un contexto escolar en el campo de la electrostática. Hemos clasificado las respuestas (Tabla $V$ ) en dos categorías. La categoría $A$ (correcta) se basa en comprender que el vector intensidad de campo sólo depende de la carga que crea el campo y no de las cargas sobre las que actúa. La categoría $B$ confunde el vector intensidad de campo y el vector fuerza eléctrica. 
Tabla V

Porcentajes de respuesta obtenidos al explicar y dibujar el vector intensidad de campo generado por una carga puntual en un punto del espacio.

\begin{tabular}{|c|c|c|c|c|}
\hline \multirow[b]{2}{*}{$\begin{array}{l}\text { Caregoria } \\
\text { de respuesta } \\
\text { del ítem } 6\end{array}$} & \multicolumn{4}{|c|}{ Porcentaje de respuesta (\%) } \\
\hline & $\begin{array}{c}3^{\circ} \mathrm{de} \text { BUP } \\
\mathrm{N}=61\end{array}$ & $\begin{array}{l}\mathrm{COU} \\
\mathrm{N}=60\end{array}$ & $\begin{array}{c}1^{\circ} \mathrm{d} e \text { Fisicas } \\
\mathrm{N}=60\end{array}$ & $\begin{array}{c}3^{\circ} \text { de Físicas } \\
N=64\end{array}$ \\
\hline $\begin{array}{l}\text { A. Distingue los vectores } \\
\text { intensidad de campo y } \\
\text { fuerza eléctrica* }\end{array}$ & 18 & 11 & 37 & 64 \\
\hline $\begin{array}{l}\text { B. Confunde los vectores } \\
\text { intensidad de campo y } \\
\text { fuerza eléctrica }\end{array}$ & 38 & 77 & 46 & 22 \\
\hline Incodificables & 23 & 2 & 5 & - \\
\hline No contesta & 21 & 10 & 12 & 14 \\
\hline
\end{tabular}

* Respuesta correcta

Al ser una pregunta de tipo operativo contestan correctamente los más familiarizados con el contexto teórico, notándose una influencia positiva del currículo. Sin embargo, es grave constatar que la confusión entre los conceptos de intensidad de campo eléctrico y fuerza eléctrica es mayoritaria hasta llegar a $3^{\circ}$ de Físicas.
En la tabla VI se muestran las respuestas de los estudiantes sobre la instantaneidad de la interacción de dos cargas puntuales separadas (ítem 7). El análisis correcto de la pregunta (no instantaneidad de las interacciones eléctricas) requiere utilizar una concepción de campo eléctrico de acción "paso a paso» frente a una concepción coulombiana de acción a distancia.

Tabla VI

Porcentajes de respuesta obtenidos al explicar la simultaneidad de la interacción entre dos cargas puntuales.

\begin{tabular}{|c|c|c|c|c|}
\hline \multirow[b]{2}{*}{$\begin{array}{l}\text { Categoria } \\
\text { de respuesta } \\
\text { del ítem } 7\end{array}$} & \multicolumn{4}{|c|}{ Porcentaje de respuesta (\%) } \\
\hline & $\begin{array}{c}3^{\circ} \text { de BUP } \\
N=61\end{array}$ & $\begin{array}{l}\mathrm{COU} \\
\mathrm{N}=60\end{array}$ & $\begin{array}{c}3^{\circ} \text { de Físicas } \\
N=60\end{array}$ & $\begin{array}{c}3^{\circ} \text { de Físicas } \\
N=64\end{array}$ \\
\hline «Es instantánea» & 54 & 63 & 56 & 31 \\
\hline «No es instantánea» & 15 & 15 & is & 44 \\
\hline Razonando correctamente* & 0 & 0 & 4 & 44 \\
\hline No lo sé & 31 & 22 & 29 & Is \\
\hline
\end{tabular}

* Respuesta correcta 
En el porcentaje de respuestas correctas, se nota una influencia positiva de la instrucción, llegándose en el nivel más alto a que $2 / 5$ de los estudiantes la interpreten como una "acción paso a paso" (teoría del campo eléctrico). Sin embargo, el número de alumnos que piensan «de forma coulombiana» es mayoritario hasta $3^{\circ}$ de Físicas, e incluso en este nivel existe $1 / 3$ de estudiantes que dan esta respuesta.

Cuando los estudiantes se enfrentan a una cuestión de conflicto cognitivo serio (ítem 8), como es la de una jaula de Faraday, el número de respuestas correctas es muy bajo incluso en niveles universitarios (Tabla VII). El análisis de la situación planteada requiere una concepción de la interacción eléctrica que se apoya en el concepto de campo eléctrico y, consecuentemente, en Ia aplicación del teorema de Gauss. También cabe la posibilidad más complicada de aplicar la ley de Coulomb a elementos de superficie cargada del cilindro y posteriormente integrarlos para toda la superficie. Esta posibilidad no ha aparecido en ninguna de las respuestas analizadas. Sin embargo, la respuesta mayoritaria consiste en una aplicación errónea de la ley de Coulomb (categoría $B$, coulombiana), donde se considera cada lado de la pared del cilindro como una carga puntual y se aplica la regla de que a menor distancia, mayor atracción. De acuerdo con los resultados, es claro que los estudiantes (incluidos los universitarios) no utilizan de forma significativa el concepto de campo eléctrico en un contexto electrostático.

Las categorías establecidas en las respuestas del cuestionario son convergentes con las del análisis de las entrevistas sobre la «jaula de Faraday», tal como se observa en los siguientes ejemplos:

1. Ejemplo de respuesta incoherente que busca una respuesta rápida sin tener en cuenta el marco teórico estudiado en clase (Julián, $3^{\circ}$ de BUP)
Entrevistador: ¿Por qué la lámina de fuera es repelida y Ia de dentro se queda vertical?

Alumno: Al doblar la lámina se cierra el circuito, y entonces la carga se queda distribuida por fuera de la superficie. Entonces, dentro del cilindro no hay ni atracción nì repulsión.

\section{E: ¿Qué entiendes por cerrarse el circuito?}

A: Bueno, no sé. Yo creo que, si no es atraída la lámina de dentro, es porque no hay carga dentro, pero fuera sí.

\section{Ejemplo de respuesta clasificada en la categoria coulombiana (Alberto, $3^{\circ}$ de BUP)}

Entrevistador: ¿Por qué la lámina de fuera es repelida y la de dentro se queda vertical?

Alumno: El papel de fuera tiene la carga en un lado, en cambio el de dentro la tiene alrededor. Entonces, todas la fuerzas que le hacen al de dentro se anulan entre sí, y se queda vertical.

E: Pero el papel no está colocado en el centro del cilindro. ¿No tendrías que tener en cuenta la distancia a la hora de calcular las fuerzas?

A: Bueno sí, pero en este caso ya se ve que se queda quieta, o sea, que se anulan.

E: Pero, ¿no es una contradicción?

A: No sé, el hecho es que sale así.

3. Ejemplo de respuesta clasificada en la categoría coulombiana (Izaskun, COU)

Entrevistador: ¿Por qué la lámina de fuera es repelida y la de dentro se queda vertical?

Tabla VII

Porcentajes de respuesta obtenidos al explicar la no-influencia eléctrica en el interior de una jaula de Faraday.

\begin{tabular}{|c|c|c|c|c|}
\hline \multirow[b]{2}{*}{$\begin{array}{l}\text { Categoría } \\
\text { de respuesta } \\
\text { del ítem } 8\end{array}$} & \multicolumn{4}{|c|}{ Porcentaje de respuesta $(\%)$} \\
\hline & $\begin{array}{c}3^{\circ} \text { de BUP } \\
N=6 I\end{array}$ & $\begin{array}{l}\mathrm{COU} \\
\mathrm{N}=60\end{array}$ & $\begin{array}{c}1^{\circ} \text { de Físicas } \\
\qquad N=60\end{array}$ & $\begin{array}{c}3^{\circ} \text { de Físicas } \\
N=64\end{array}$ \\
\hline No hay interacción (A)* & - & - & 10 & 14 \\
\hline Sí hay interacción (B) & 97 & 87 & 75 & 81 \\
\hline No contesta & 3 & 13 & I5 & 5 \\
\hline
\end{tabular}

\footnotetext{
* Respuesta correcta
} 
Alumna: Cuando cierras la lámina debería seguir repeliéndose, pero, como por el otro lado también tienes fuerza, pues se contrarrestan, ¿no?

E: Pero al no estar el papel en el centro, ¿las fuerzas de un lado no serán mayores que las del otro debido a la diferencia de distancias?

A: Bueno, no sé. Pero aquí se ve que se anulan y el papel se queda vertical.

\section{Ejemplo de respuesta correcta (Dori, $1^{\circ}$ de Físicas)}

Entrevistador: ¿Por qué la lámina de fuera es repelida y la de dentro se queda vertical?

Alumna: Porque dentro del conductor no tiene que haber campo, bueno, eso... es porque dentro de... esto es como un conductor, la lámina de metal; entonces, tú al cerrarlo, haces como si fuera una superficie cerrada, entonces... por Gauss, dice que dentro de una superficie metálica cerrada el campo es cero... entonces, fuera sí hay campo pero dentro no, por eso la lámina de papel de dentro no experimenta ninguna fuerza y la de fuera sí.

\section{CONCLUSIONES}

Los resultados obtenidos parecen indicar que la mayoría de los estudiantes asumen la naturaleza eléctrica de la materia (Ítem 1), aunque existe un porcentaje minoritario y constante a lo largo de la intrucción que aboga por la «creación de cargas» en los fenómenos triboeléctri$\cos$. De acuerdo con la primera hipótesis, se comprueba que aquellos fenómenos eléctricos (ítem 3 ) que puedan ser explicados fácilmente por un modelo hidrostático de carga eléctrica y que requieren un conocimiento procedimental de «sentido común» tienen alto porcentaje de éxito entre los estudiantes. Sin embargo, de acuerdo con la gradación epistemológica indicada en el apartado 3, aparecen problemas a la hora de interpretar fenomenos de no-electrización de un metal por frotamiento (Ítem 2), fenómenos de inducción (Ítem 4) y, en particular, aquellas situaciones donde la explicación requiera considerar el papel desempeñado por el medio (Item 5). En estos casos, aparecen también dificultades de tipo procedimental o estratégico como la fijación funcional (Ítem 5), el causalismo simple (Ítem 2) o la inversión de la implicación causa-efecto (Item 4).

Asimismo, y de acuerdo con la segunda hipótesis, Ios resultados parecen confirmar que existe cierto paralelis- mo entre los problemas habidos en la construcción de la electricidad y las dificultades de aprendizaje de los estudiantes. En efecto, los problemas epistemológicos que tuvo que superar la ciencia para llegar a construir una teoría del campo eléctrico (proceso de unificación de la teoría relativa a las cinco clases de electricidad y del magnetismo conocidas en tiempos de Faraday) que fuera más allá del nivel de formulación coulombiano implican una síntesis de conocimientos y alto grado de abstracción que permite explicar las grandes dificultades que tienen los estudiantes en estas teorías. No obstante, y como también indica la investigación sobre concepciones alternativas, las preconcepciones de los estudiantes pueden interaccionar con el conocimiento científico que trata de enseñar el profesor en clase, y pueden resultar adaptaciones imprevistas que se traducen en modelos de explicación sui generis que obviamente no se han constatado en el desarrollo histórico (p.e., la categoría A.2 del ítem 1). Asimismo, se ha contrastado que las dificultades conceptuales indicadas anteriormente van acompañadas de nuevas dificultades procedimentales (razonamientos de «sentido común», causalidad simple, inversión de la implicación causa-efecto), dado que no se ha familiarizado a los estudiantes con aspectos esenciales de la metodología científica.

Por otro lado, y de acuerdo con los resultados obtenidos, podemos decir que muy pocos estudiantes de secundaria y universidad «aceptan», es decir, utilizan de forma significativa el concepto de campo eléctrico, confirmando la tercera hipótesis realizada. Respecto de esta hipótesis, los resultados parecen confirmar que los estudiantes no establecen una diferenciación neta entre los conceptos de intensidad de campo y fuerza eléctrica, incluso en situaciones familiares dentro de un contexto escolar (Ítem 6). Es más, cuando se presentan situaciones de conflicto cognitivo que requieran una aplicación significativa del concepto de campo eléctrico (Ítems 7 y 8 ), se eleva aún más el porcentaje de respuestas erróneas.

Si a las dificultades conceptuales y epistemológicas señaladas añadimos los efectos de una enseñanza basada en una visión exclusivamente analítica del trabajo científico (que resalta la necesaria parcialización de los estudios, pero que olvida los esfuerzos posteriores de unificación y de construcción de cuerpos coherentes de conocimientos cada vez más inclusores), podemos explicar las grandes dificultades que tienen los estudiantes en estas teorías. Así pues, serấ necesario idear estrategias constructivistas de enseñanza que puedan facilitar un cambio conceptual, epistemológico y metodológico. Esta cuestión será objeto de próximos trabajos. 


\section{REFERENCIAS BIBLIOGRÁFICAS}

ANDERSON, J.R. (1990). Cognitive Psychology and its implications. Nueva York: W.H. Freeman and company.

BACHELARD, G. (1940). La philosophie du non. Essai d' une philosophie dunouvel esprit scientifique. Presses Universitaires de France. París. Trad. cast., 1984. Buenos Aires: Amorrortu editores.

BERKSON, W. (1981). Las teorias de los campos de fuerza desde Faraday hasta Einstein. Madrid: Alianza Editorial.

BURBULES, N. y LINN, M. (1991). Science education and phylosophy of science:congruence or contradiction? International Journal of Science Education, 13(3), pp. 227$24 i$.

DUIT, R. (1993). Research on student's conceptions-developments and trends, paper presented at the «Third International Seminar on Misconceptions and Educational Strategies in Science and Mathematics». Cornell. Ithaca. EEUU.

DUSCH, R.A. (1995). Más allá del conocimiento: los desafíos epistemológicos y sociales de la enseñanza mediante cambio conceptual. Enseñanza de las Ciencias, 13(1), po. 3-14.

EYLON, 8. y GANIEL, U. (1990). Macro-micro relationships: the missing link between electrostatics and electrodynamics in students' reasoning. International Journal of Science Education, 12,(1), pp. 79-94.

FERREIRA, N. (1987). Activités en électrostatique. Bolletin de l'Union des Physiciens, 713, pp. 477-490.

FURIO, C. (1996). Dificultades procedimentales en el aprendizaje de la química: Ia fijación y la reducción funcionales. Aspectos didácticos de la física y la química. ICE. Universidad de Zaragoza (en prensa).

FURIÓ, C. (1994). La enseñanza de las ciencias como investigación: un modelo emergente. Proceedings Intemational Conference "Science and Mathematics Education for the 21 st. Century: Towards innovatory approaches». Concepción. Chile: Universidad de Concepción.

FURIÓ, C., HERNÁNDEZ, J. y HARRIS, H. (1987). Parallels between adolescent's concepcion of gases and History of Chemistry. Journal of Chemical Education, 64(7), pp. 616-618.

GALILI, I. (1995). Mechanics background influences students'conceptions in electromagnetism. International Journal of Science Education, 17(3), pp. 371-387.

GIANNETTO, E., TARSITANI, C. y VICENTINI, M. (1992). The relations between Epistemology, History of Science and Science Teaching from the point of view of the Research on Mental Representations. Proceedings of the Internacional Conference «History \& Philosophy of Science Education», pp. 359-374. Kingston: Queen's University.
GIL, D. y CARRASCOSA, I. (1985). Science learning as a conceptual and methodological change. European Journal of Science Education, 5, pp. 70-81.

GIL, D., CARRASCOSA, J, FURIÓ, C. y MARTÍNEZTORREGROSA, J. (1991). La enseñanza de las ciencias en la educación secundaria. Barcelona: ICE de la Universidad de Barcelona-Horsori.

HEILBRON, J.L. (1979). Electricity in the $17^{\text {th }}$ and $18^{\text {th }}$ centuries. Berkeley and Los Angeles. California: University of California Press.

MATTHEWS, M.R. (1990). History, Philosophy and Science Teaching: A rapprochement. Studies in Science Education, 18, pp. 25-51.

MINISTERIO DE EDUCACIÓN Y CIENCIA (MEC). (1991). Bachillerato: estructura y contenidos, pp. 175-190.

MORTIMER, E.F. (1995). Conceptual change or conceptual profile change. Science \& Education, 4, pp. 267-285.

NUSSBAUM,J.(1983). Clasroom conceptual change: the lesson to be leamed from the history of science. Proceedings of the International Seminar on Misconceptions in Science and Mathematics, pp. 272-281, en Helm, H. y Novack, J.D. (eds.). Nueva York: Department of Education. Comell University.

SALTIEL, E. y VIENNOT, L. (1985). ¿Qué aprendemos de las semejanzas entre las ideas históricas y el razonamiento espontáneo de los estudiantes? Enseñanza de las Ciencias, 3(2), pp. 137-144

TATON, R. (1985). Histoire Generale des Sciences. Presses Universitaires de France. París. Trad. cast., 1988. Barcelona: Ediciones Orbis, SA.

TOULMIN, S. (1972). Human understanding, $1:$ The Collective use and evolution of concepts. Princeton University Press. Trad. cast., 1977. Madrid: Alianza Editorial.

THUILLIER, $P$. (1989). De I fillosofía al electromagnetismo: el caso Oersted. Mundo Cientifico, 10, p. 102.

VIENNOT, L. y RAINSON, S. (1992). Students' reasoning about the superposition of electric fields. International Journal of Science Education, 14(4), pp. 475.487.

WANDERSEE, J.H. (1992). The Historicality of cognition: implications for Science Education Research. Journal of Research in Science Education, 29 (4), pp. 423-434.

WANDERSEE J.H., MINTZES J.J. y NOVAK, J.D. (1994). Research on alternative conceptions in Science. Handbook of research on Science teaching and learning. Nueva York: McMillan Publishing Company.

WHITTAKER, E. (1987). A History of the Theories of Aether and Electricity, Vol. I. Tomash Publishers. American Institute of Physics. 


\section{ANEXO I}

Item 1. Se frota con un paño de lana una barra de plástico, quedando la barra electrizada. Explica esta electrización y dibuja (en la figura $b$ cómo te imaginas que estaría distribuida la carga en la barra electrizada.

$a$

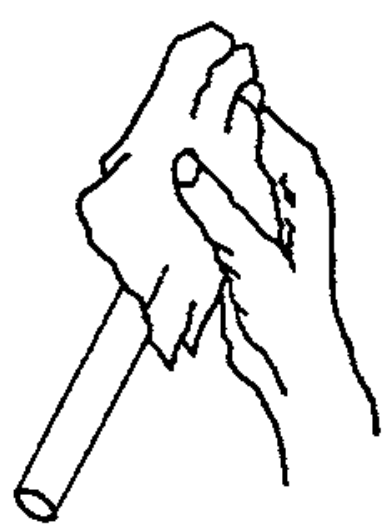

$b$

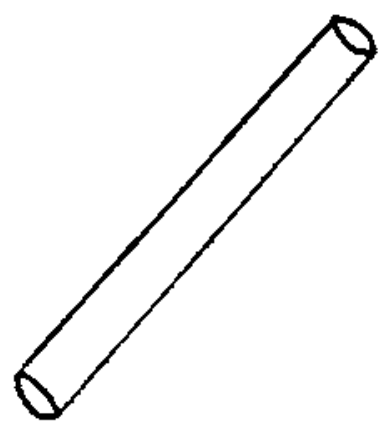

ftem 2. Al frotar con un paño de lana una barra metálica, se comprueba que ésta no queda electrizada. ¿Cómo se explica que no se electrice la barra?

Item 3. Con un bolígrafo cargado se toca una bolita de corcho. Al cabo de unos instantes se observa que el bolígrafo y la bolita se repelen. Explica cómo puede producirse esta repulsión.

Item 4. Un bolígrafo frotado es capaz de atraer un trocito de papel próximo a él. Explica cómo se produce esta interacción.

f́tem 5. Se aproxima una hoja de plástico cargada al extremo de una barra larga de madera sin tocaria, como se vê en la figura. En el otro extremo de la barra hay una bolita de polturetano. Explica si seráa atraida o no la bolita.
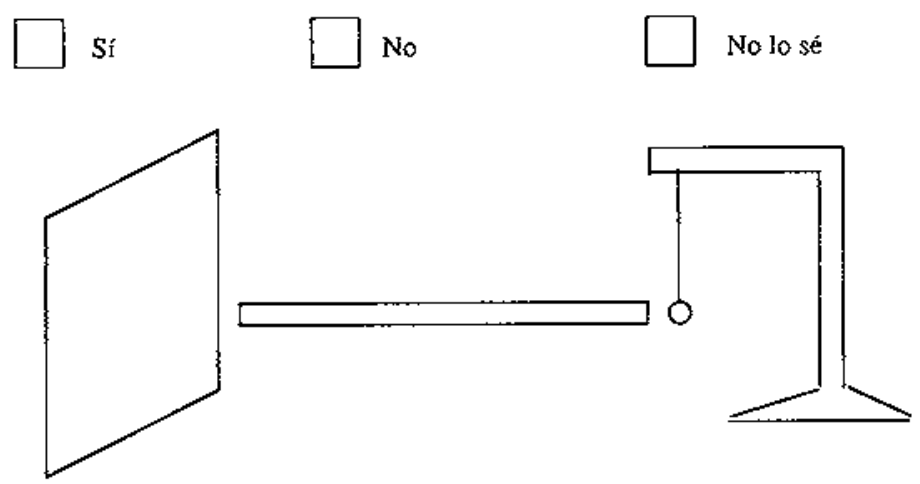

Item 6. En el punto $P$ del espacio próximo a $Q$ (Fig. 6a) se ha representado el valor de la intensidad del campo $E$ en dicho punto. ¿Cual sería la representación que le correspondería a la intensidad del campo eléctrico en ese punto si se coloca allí una carga negativa muy pequeña (Fig. 6b). Dibújala y explica tu contestación.
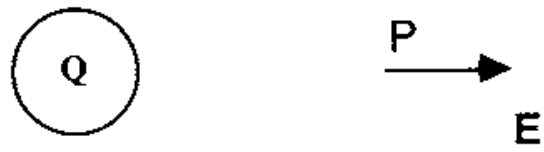

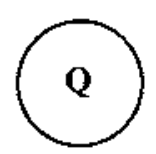

$\stackrel{P}{\ominus}$

q

$6 a$

$6 b$ 
Ítem 7. A una cierta distancia de una carga $Q$ se coloca otra carga pequeña $q$. ¿Las fuerzas que se ejercen ambas cargas aparecerán en el mismo instante en que se ha colocado $q$, o después de haber pasado un breve tiempo? Explica la respuesta.

Ítem 8. La figura muestra un bote metálico cargado negativamente. ¿Qué sucederá al introducir un péndulo cargado positivamente dentro del mismo? Véase figura.

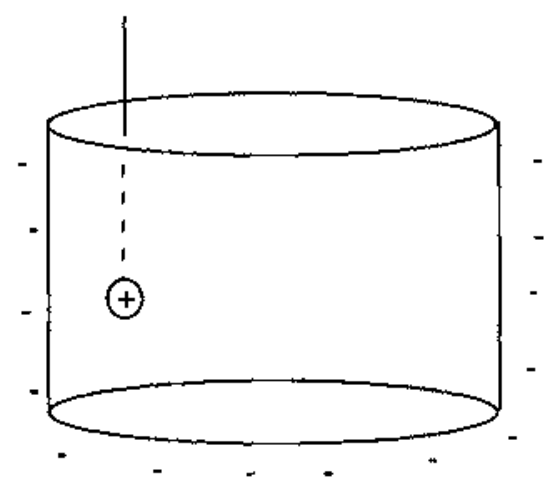

